\title{
New Fixed Point Theorems of Mixed Monotone Operators and Applications to Singular Boundary Value Problems on Time Scales
}

\author{
Huiye Xu \\ College of Economics and Management, North University of China, Taiyuan, Shanxi 030051, China \\ Correspondence should be addressed to Huiye Xu, silviahsu2005@yahoo.com.cn
}

Received 3 July 2010; Accepted 13 December 2010

Academic Editor: Daniel Franco

Copyright (c) 2011 Huiye $\mathrm{Xu}$. This is an open access article distributed under the Creative Commons Attribution License, which permits unrestricted use, distribution, and reproduction in any medium, provided the original work is properly cited.

Some new existence and uniqueness theorems of fixed points of mixed monotone operators are obtained, and then they are applied to a nonlinear singular second-order three-point boundary value problem on time scales. We prove the existence and uniqueness of a positive solution for the above problem which cannot be solved by using previously available methods.

\section{Introduction}

The study of mixed monotone operators has been a matter of discussion since they were introduced by Guo and Lakshmikantham [1] in 1987, because it has not only important theoretical meaning but also wide applications in microeconomics, the nuclear industry, and so on (see [1-4]). Recently, some new and interesting results about these kinds of operators have emerged, and they are used extensively in nonlinear differential and integral equations (see [5-9]).

In this paper, we extend the main results of [9] to mixed monotone operators. Without demanding compactness and continuity conditions and the existence of upper and lower solutions, we study the existence, uniqueness, and iterative convergence of fixed points of a class of mixed monotone operators. Then, we apply these results to the following singular second-order three-point boundary value problem on time scales:

$$
\begin{gathered}
-x^{\prime \prime}(t)=w(t)\left[f_{1}(x(t))+f_{2}(x(t))\right], \quad t \in[a, b]_{\mathbb{T}}, \\
x(a)=0, \quad x\left(\sigma^{2}(b)\right)=\delta x(\eta),
\end{gathered}
$$


where $a, b \in \mathbb{T}$ with $a<b, \eta \in(a, b)_{\mathbb{T}}$, and $0<\delta<\left(\sigma^{2}(b)-a\right) /(\eta-a)$. The functions $w:(a, b) \rightarrow[0,+\infty)$ and $f_{i}:(0,+\infty) \rightarrow[0,+\infty)(i=1,2)$ are continuous. Our nonlinearity $w$ may have singularity at $t=a$ and / or $t=b$ and $f_{i}(x)(i=1,2)$ may have singularity at $x=0$.

To understand the notations used in (1.1), we recall that $\mathbb{T}$ is a time scales, that is, $\mathbb{T}$ is an arbitrary nonempty closed subset of $\mathbb{R}$. For each interval $I$ of $\mathbb{R}$, we define $I_{\mathbb{T}}=I \cap \mathbb{T}$. For more details on time scales, one can refer to [10-12].

In recent years, there is much attention paid to the existence of positive solutions for nonlocal boundary value problems on time scales, see [13-18] and references therein. Dynamic equations have been applied in the study of insect population models, stock market and heat transfer and so on [19-22]. Time scales can be used in microeconomics models to study behavior which is sometimes continuous and sometimes discrete. A simple example of this continuous-discrete behavior is seen in suppliers short-run decisions and long-run decisions. Unifying both continuous and discrete model can avoid repeat research and has the capacity to get some different types of models which neither continuous models nor discrete models can effectively describe.

On the other hand, singular boundary value problems on time scales have also been investigated extensively (see [23-27]). We would like to mention some results of DaCunha et al. [23], Hao et al. [25], Luo [26], and Hu [27], which motivated us to consider problem (1.1).

In [23], DaCunha et al. considered the following singular second-order three-point dynamic boundary value problem:

$$
\begin{aligned}
& -x^{\prime \prime}(t)=f(t, x), \quad t \in(0,1]_{\mathbb{T}}, \\
& x(0)=0, \quad x\left(\sigma^{2}(1)\right)=x(\eta),
\end{aligned}
$$

where $\eta \in(0,1]_{\mathbb{T}}$ is fixed and $f(t, x)$ is singular at $x=0$ and possible at $t=0, x=\infty$. The authors claimed that "we note that this is the first work (to our knowledge) that deals with singular boundary value problems in a general time scales setting." The results on existence of positive solutions were obtained by means of a fixed point theorem due to Gatica, Oliker and Waltman for mappings that are decreasing with respect to a cone.

In [25], Hao et al. were concerned with the following singular boundary value problem of nonlinear dynamic equation

$$
\begin{aligned}
& {\left[\varphi(t) x^{\Delta}(t)\right]^{\Delta}+\lambda m(t) f(t, x(\sigma(t)))=0, \quad t \in[a, b]_{\mathbb{T}},} \\
& \alpha x(a)-\beta x^{\Delta}(a)=0, \quad \gamma x(\sigma(b))+\delta x^{\Delta}(\sigma(b))=0,
\end{aligned}
$$

where $m(\cdot):(a, \sigma(b))_{\mathbb{T}} \rightarrow[0,+\infty)$ is rl-continuous and may be singular at $t=a$ and $/$ or $t=\sigma(b)$. With suitable growth and limit conditions, an existence theorem of positive solutions was established by using the Krasnoselskii fixed point theorem. 
In [26], Luo studied the following singular $m$-point dynamic eigenvalue problem with mixed derivatives:

$$
\begin{gathered}
-\left(p(t) u^{\Delta}(t)\right)^{\nabla}=\lambda f(t, u(t)), \quad t \in(0,1]_{\mathbb{T}}, \\
u(0)=\sum_{i=1}^{m-2} a_{i} u\left(\xi_{i}\right), \quad \gamma u(1)+\delta p(1) u^{\Delta}(1)=\sum_{i=1}^{m-2} b_{i} p\left(\xi_{i}\right) u^{\Delta}\left(\xi_{i}\right),
\end{gathered}
$$

where $f(t, w)$ is singular at $t=0$ and $w=0$. The author obtained eigenvalue intervals in which there exists at least one positive solution of problem (1.4) by making use of the fixed point index theory.

In [27], $\mathrm{Hu}$ were concerned with the following singular third-order three-point boundary value problem on time scales:

$$
\begin{gathered}
\left(u^{\Delta \Delta}(t)\right)^{\nabla}+w(t) f(t, u(t))=0, \quad t \in[a, b]_{\mathbb{T}}, \\
u(\rho(a))-\beta u^{\Delta}(\rho(a))=\alpha u(\eta), \quad \gamma u(\eta)=u(b), \quad u^{\Delta \Delta}(\rho(a))=0,
\end{gathered}
$$

where $w:(a, b)_{\mathbb{T}} \rightarrow[0,+\infty)$ and $f:[a, b]_{\mathbb{T}} \times(0,+\infty) \rightarrow[0,+\infty)$ are continuous. The nonlinearity $w$ may have singularity at $t=a$ and /or $t=b$ and $f(t, u)$ may have singularity at $u=0$. With the aid of the fixed point theorem of cone expansion and compression type, results on the existence of positive solutions to (1.5) were obtained in the bounded set.

From the above research, we note that there is no result on the uniqueness of solutions and convergence of the iterative sequences for singular boundary value problems on time scales. As we know, completely continuity condition is crucial for the above discussion. However, it is difficult to verify for singular problems on time scales, in particular, in order to remove the singularity in $f(u)$ at $u=0$, more restricted conditions are required. For instance, condition $\left(A_{1}\right)$ of Theorem 2.3 in [23] and condition $\left(C_{2}\right)$ of Theorem 3.1 in [27]. In our abstract results on mixed monotone operators, since the compactness and continuity conditions are not required, they can be directly applied to singular boundary value problem (1.1).

The purpose of this paper is to present some conditions for problem (1.1) that have a unique solution, the iterative sequences yielding approximate solutions are also given. Our main result generalizes and improves Theorem 2.3 in [18].

\section{Preliminaries and Abstract Theorems}

Let the real Banach space $E$ be partially ordered by a cone $P$ of $E$, that is, $x \leq y$ if and only if $y-x \in P$. $A: P \times P \rightarrow P$ is said to be a mixed monotone operator if $A(x, y)$ is increasing in $x$ and decreasing in $y$, that is, $u_{i}, v_{i}(i=1,2) \in P, u_{1} \leq u_{2}, v_{1} \geq v_{2}$ implies $A\left(u_{1}, v_{1}\right) \leq A\left(u_{2}, v_{2}\right)$. Element $x \in P$ is called a fixed point of $A$ if $A(x, x)=x$.

Recall that cone $P$ is said to be solid if the interior $P^{\circ}$ is nonempty and we denote $x \gg \theta$ if $x \in P^{\circ} . P$ is said to be normal if there exists a positive constant $N$, such that $\theta \leq x \leq y \Rightarrow$ $\|x\| \leq N\|y\|$, the smallest $N$ is called the normal constant of $P$. For all $x, y \in E$, the notation 
$x \sim y$ means that there exist $\lambda>0$ and $\mu>0$ such that $\lambda x \leq y \leq \mu x$. Clearly, $\sim$ is an equivalence relation. Given $h>\theta$ (i.e., $h \geq \theta$ and $h \neq \theta$ ), we denote by $P_{h}$ the set $P_{h}=\{x \in E \mid x \sim h\}$. It is easy to see that $P_{h} \subset P$ is convex and $\lambda P_{h}=P_{h}$ for all $\lambda>0$. If $P^{\circ} \neq \emptyset$ and $h \in P^{\circ}$, it is clear that $P_{h}=P^{\circ}$.

All the concepts discussed above can be found in [1, 2, 4]. For more results about mixed monotone operators and other related concepts, the reader is referred to [3,5-9] and some of the references therein.

In [9], Zhai and Cao introduced the following definition of $\tau-\varphi$-concave operators.

Definition 2.1 (see [9]). Let $E$ be a real Banach space and $P$ be a cone in $E$. We say an operator $A: P \rightarrow P$ is $\tau$ - $\varphi$-concave if there exist two positive-valued functions $\tau(t), \varphi(t)$ on interval $(a, b)$ such that

$$
\begin{aligned}
& \left(H_{1}\right) \tau:(a, b) \rightarrow(0,1) \text { is a surjection; } \\
& \left(H_{2}\right) \varphi(t)>\tau(t), \text { for all } t \in(a, b) \\
& \left(H_{3}\right) A(\tau(t) x) \geq \varphi(t) A x, \text { for all } t \in(a, b), x \in P .
\end{aligned}
$$

They obtained the following result.

Theorem 2.2 (see [9]). Let E be a real Banach space and $P$ be a normal cone in E. Suppose that an operator $A: P \rightarrow P$ is increasing and $\tau$ - $\varphi$-concave. In addition, suppose that there exists $h \in P \backslash\{\theta\}$ such that $A h \in P_{h}$. Then

(i) there are $u_{0}, v_{0} \in P_{h}$ and $r \in(0,1)$ such that $r v_{0} \leq u_{0} \leq v_{0}, u_{0} \leq A u_{0} \leq A v_{0} \leq v_{0}$;

(ii) operator A has a unique fixed point $x^{*}$ in $\left[u_{0}, v_{0}\right]$;

(iii) for any initial $x_{0} \in P_{h}$, constructing successively the sequence $x_{n}=A x_{n-1}, n=1,2, \ldots$, we have $\left\|x_{n}-x^{*}\right\| \rightarrow 0(n \rightarrow \infty)$.

We can extend Theorem 2.2 to mixed monotone operators, our main results can be stated as follows.

Theorem 2.3. Let $P$ be a normal cone in a real Banach space $E$, and $A: P \times P \rightarrow P$ a mixed monotone operator. Assume that for all $a<t<b$, there exist two positive-valued functions $\tau(t), \varphi(t)$ on interval $(a, b)$ such that

$\left(C_{1}\right) \tau:(a, b) \rightarrow(0,1)$ is a surjection;

$\left(C_{2}\right) \varphi(t)>\tau(t)$, for all $t \in(a, b)$;

$\left(C_{3}\right) A(\tau(t) x,(1 / \tau(t)) y) \geq \varphi(t) A(x, y)$, for all $t \in(a, b), x, y \in P$.

In addition, suppose that there exists $h \in P \backslash\{\theta\}$ such that $A(h, h) \in P_{h}$. Then

(i) there are $u_{0}, v_{0} \in P_{h}$ and $r \in(0,1)$ such that $r v_{0} \leq u_{0} \leq v_{0}, u_{0} \leq A\left(u_{0}, v_{0}\right) \leq A\left(v_{0}, u_{0}\right) \leq$ $v_{0}$;

(ii) operator A has a unique fixed point $x^{*}$ in $\left[u_{0}, v_{0}\right]$;

(iii) for any initial $x_{0}, y_{0} \in P_{h}$, constructing successively the sequences $x_{n}=A\left(x_{n-1}, y_{n-1}\right)$, $y_{n}=A\left(y_{n-1}, x_{n-1}\right), n=1,2, \ldots$, we have $\left\|x_{n}-x^{*}\right\| \rightarrow 0$ and $\left\|y_{n}-x^{*}\right\| \rightarrow 0$ as $n \rightarrow \infty$. 
Corollary 2.4. Let E be a real Banach space, $P$ a normal, solid cone in E. Suppose $A: P^{\circ} \times P^{\circ} \rightarrow P^{\circ}$ is a mixed monotone operator and satisfies the conditions $\left(C_{1}\right)-\left(C_{3}\right)$ of Theorem 2.3. Then

(i) there are $u_{0}, v_{0} \in P^{\circ}$ and $r \in(0,1)$ such that $r v_{0} \leq u_{0} \leq v_{0}, u_{0} \leq A\left(u_{0}, v_{0}\right) \leq A\left(v_{0}, u_{0}\right) \leq$ $v_{0}$;

(ii) operator A has a unique fixed point $x^{*}$ in $P^{\circ}$;

(iii) for any initial $x_{0}, y_{0} \in P^{\circ}$, constructing successively the sequences $x_{n}=A\left(x_{n-1}, y_{n-1}\right)$, $y_{n}=A\left(y_{n-1}, x_{n-1}\right), n=1,2, \ldots$, we have $\left\|x_{n}-x^{*}\right\| \rightarrow 0$ and $\left\|y_{n}-x^{*}\right\| \rightarrow 0$ as $n \rightarrow \infty$.

Remark 2.5. In Theorem 2.3, if $A: P^{\circ} \times P^{\circ} \rightarrow P^{\circ}$ with $P$ is a solid cone, we can know that $A(h, h) \in P_{h}$ is automatically satisfied. Therefore, we can deduce that Corollary 2.4 holds from Theorem 2.3. For simplicity, we only present the proof of Theorem 2.3.

Proof of Theorem 2.3. Note that $A(h, h) \in P_{h}$, we can find a sufficiently small number $\bar{e}_{0} \in(0,1)$ such that

$$
\bar{e}_{0} h \leq A(h, h) \leq \frac{h}{\bar{e}_{0}}
$$

According to $\left(C_{1}\right)$, we can obtain that there exists $t_{1} \in(a, b)$ such that $\tau\left(t_{1}\right)=\bar{e}_{0}$, thus

$$
\tau\left(t_{1}\right) h \leq A(h, h) \leq \frac{h}{\tau\left(t_{1}\right)} .
$$

Since $\varphi\left(t_{1}\right)>\tau\left(t_{1}\right)$, we can find a positive integer $k$ such that

$$
\left(\frac{\varphi\left(t_{1}\right)}{\tau\left(t_{1}\right)}\right)^{k} \geq \frac{1}{\tau\left(t_{1}\right)}
$$

Let $u_{0}=\left[\tau\left(t_{1}\right)\right]^{k} h, v_{0}=\left(1 /\left[\tau\left(t_{1}\right)\right]^{k}\right) h$, and construct successively the sequences

$$
u_{n}=A\left(u_{n-1}, v_{n-1}\right), \quad v_{n}=A\left(v_{n-1}, u_{n-1}\right), \quad n=1,2, \ldots
$$

It is clear that $u_{0}, v_{0} \in P_{h}$ and $u_{0}<v_{0}, u_{1}=A\left(u_{0}, v_{0}\right) \leq A\left(v_{0}, u_{0}\right)=v_{1}$. In general, we obtain $u_{n} \leq v_{n}, n=1,2, \ldots$

It follows from $\left(C_{3}\right),(2.2)$, and (2.3) that

$$
\begin{aligned}
u_{1} & =A\left(u_{0}, v_{0}\right)=A\left(\left[\tau\left(t_{1}\right)\right]^{k} h, \frac{h}{\left[\tau\left(t_{1}\right)\right]^{k}}\right) \\
& =A\left(\tau\left(t_{1}\right)\left[\tau\left(t_{1}\right)\right]^{k-1} h, \frac{1}{\tau\left(t_{1}\right)} \frac{h}{\left[\tau\left(t_{1}\right)\right]^{k-1}}\right) \\
& \geq \varphi\left(t_{1}\right) A\left(\left[\tau\left(t_{1}\right)\right]^{k-1} h, \frac{h}{\left[\tau\left(t_{1}\right)\right]^{k-1}}\right)
\end{aligned}
$$




$$
\begin{aligned}
& =\varphi\left(t_{1}\right) A\left(\tau\left(t_{1}\right)\left[\tau\left(t_{1}\right)\right]^{k-2} h, \frac{1}{\tau\left(t_{1}\right)} \frac{h}{\left[\tau\left(t_{1}\right)\right]^{k-2}}\right) \\
& \geq\left[\varphi\left(t_{1}\right)\right]^{2} A\left(\left[\tau\left(t_{1}\right)\right]^{k-2} h, \frac{h}{\left[\tau\left(t_{1}\right)\right]^{k-2}}\right) \\
& \geq \cdots \geq\left[\varphi\left(t_{1}\right)\right]^{k} A(h, h) \\
& \geq\left[\varphi\left(t_{1}\right)\right]^{k} \tau\left(t_{1}\right) h \\
& \geq\left[\tau\left(t_{1}\right)\right]^{k} h=u_{0} .
\end{aligned}
$$

From $\left(C_{3}\right)$, we have

$$
A\left(\frac{x}{\tau(t)}, \tau(t) y\right) \leq \frac{1}{\varphi(t)} A(x, y), \quad \forall t \in(a, b), x, y \in P
$$

Combining (2.2) with (2.3) and (2.6), we have

$$
\begin{aligned}
v_{1} & =A\left(v_{0}, u_{0}\right)=A\left(\frac{h}{\left[\tau\left(t_{1}\right)\right]^{k}},\left[\tau\left(t_{1}\right)\right]^{k} h\right) \\
& =A\left(\frac{1}{\tau\left(t_{1}\right)} \frac{h}{\left[\tau\left(t_{1}\right)\right]^{k-1}}, \tau\left(t_{1}\right)\left[\tau\left(t_{1}\right)\right]^{k-1} h\right) \\
& \leq \frac{1}{\varphi\left(t_{1}\right)} A\left(\frac{h}{\left[\tau\left(t_{1}\right)\right]^{k-1}},\left[\tau\left(t_{1}\right)\right]^{k-1} h\right) \\
& =\frac{1}{\varphi\left(t_{1}\right)} A\left(\frac{1}{\tau\left(t_{1}\right)} \frac{h}{\left[\tau\left(t_{1}\right)\right]^{k-2}}, \tau\left(t_{1}\right)\left[\tau\left(t_{1}\right)\right]^{k-2} h\right) \\
& \leq\left[\frac{1}{\varphi\left(t_{1}\right)}\right]^{2} A\left(\frac{h}{\left[\tau\left(t_{1}\right)\right]^{k-2}},\left[\tau\left(t_{1}\right)\right]^{k-2} h\right) \\
& \leq \cdots \leq\left[\frac{1}{\varphi\left(t_{1}\right)}\right]^{k} A(h, h) \\
& \leq\left[\frac{1}{\varphi\left(t_{1}\right)}\right]^{k} \frac{h}{\tau\left(t_{1}\right)} \\
& \leq \frac{h}{\left[\tau\left(t_{1}\right)\right]^{k}}=v_{0} .
\end{aligned}
$$


Thus, we obtain

$$
u_{0} \leq u_{1} \leq v_{1} \leq v_{0}
$$

By induction, it is easy to obtain that

$$
u_{0} \leq u_{1} \leq \cdots \leq u_{n} \leq \cdots \leq v_{n} \leq \cdots \leq v_{1} \leq v_{0}
$$

Take any $r \in\left(0,\left[\tau\left(t_{1}\right)\right]^{2 k}\right)$, then $r \in(0,1)$ and $u_{0} \geq r v_{0}$. So we can know that

$$
u_{n} \geq u_{0} \geq r v_{0} \geq r v_{n}, \quad n=1,2, \ldots
$$

Let

$$
r_{n}=\sup \left\{r>0 \mid u_{n} \geq r v_{n}\right\}, \quad n=1,2, \ldots
$$

Thus, we have $u_{n} \geq r_{n} v_{n}, n=1,2, \ldots$, and then

$$
u_{n+1} \geq u_{n} \geq r_{n} v_{n} \geq r_{n} v_{n+1}, \quad n=1,2, \ldots
$$

Therefore, $r_{n+1} \geq r_{n}$; that is,

$$
0<r_{0} \leq r_{1} \leq \cdots \leq r_{n} \leq \cdots \leq 1
$$

Set $r^{*}=\lim _{n \rightarrow \infty} r_{n}$, we will show that $r^{*}=1$. In fact, if $0<r^{*}<1$, by $\left(C_{1}\right)$, there exists $t_{2} \in(a, b)$ such that $\tau\left(t_{2}\right)=r^{*}$. Consider the following two cases.

(i) There exists an integer $N$ such that $r_{N}=r^{*}$. In this case, we have $r_{n}=r^{*}$ and $u_{n} \geq r^{*} v_{n}$ for all $n \geq N$ hold. Hence

$$
\begin{aligned}
u_{n+1} & =A\left(u_{n}, v_{n}\right) \geq A\left(r^{*} v_{n}, \frac{1}{r^{*}} u_{n}\right)=A\left(\tau\left(t_{2}\right) v_{n}, \frac{1}{\tau\left(t_{2}\right)} u_{n}\right) \\
& \geq \varphi\left(t_{2}\right) A\left(v_{n}, u_{n}\right)=\varphi\left(t_{2}\right) v_{n+1}, \quad n \geq N .
\end{aligned}
$$

By the definition of $r_{n}$, we have

$$
r_{n+1}=r^{*} \geq \varphi\left(t_{2}\right)>\tau\left(t_{2}\right)=r^{*}, \quad n \geq N,
$$

which is a contradiction. 
(ii) For all integers $n, r_{n}<r^{*}$. Then, we obtain $0<r_{n} / r^{*}<1$. By $\left(C_{1}\right)$, there exist $z_{n} \in(a, b)$ such that $\tau\left(z_{n}\right)=r_{n} / r^{*}$. Hence

$$
\begin{aligned}
u_{n+1} & =A\left(u_{n}, v_{n}\right) \geq A\left(r_{n} v_{n}, \frac{1}{r_{n}} u_{n}\right)=A\left(\frac{r_{n}}{r^{*}} r^{*} v_{n}, \frac{1}{\left(r_{n} / r^{*}\right) r^{*}} u_{n}\right)=A\left(\tau\left(z_{n}\right) r^{*} v_{n}, \frac{1}{\tau\left(z_{n}\right) r^{*}} u_{n}\right) \\
& \geq \varphi\left(z_{n}\right) A\left(r^{*} v_{n}, \frac{1}{r^{*}} u_{n}\right)=\varphi\left(z_{n}\right) A\left(\tau\left(t_{2}\right) v_{n}, \frac{1}{\tau\left(t_{2}\right)} u_{n}\right) \\
& \geq \varphi\left(z_{n}\right) \varphi\left(t_{2}\right) A\left(v_{n}, u_{n}\right)=\varphi\left(z_{n}\right) \varphi\left(t_{2}\right) v_{n+1} .
\end{aligned}
$$

By the definition of $r_{n}$, we have

$$
r_{n+1} \geq \varphi\left(z_{n}\right) \varphi\left(t_{2}\right)>\tau\left(z_{n}\right) \varphi\left(t_{2}\right)=\frac{r_{n}}{r^{*}} \varphi\left(t_{2}\right) .
$$

Let $n \rightarrow \infty$, we have

$$
r^{*} \geq \varphi\left(t_{2}\right)>\tau\left(t_{2}\right)=r^{*}
$$

which is also a contradiction. Thus, $\lim _{n \rightarrow \infty} r_{n}=1$.

Furthermore, similarly to the proof of Theorem 2.1 in [9], there exits $x^{*} \in\left[u_{0}, v_{0}\right]$ such that $\lim _{n \rightarrow \infty} u_{n}=\lim _{n \rightarrow \infty} v_{n}=x^{*}$, and $x^{*}$ is the fixed point of operator $A$.

In the following, we prove that $x^{*}$ is the unique fixed point of $A$ in $P_{h}$. In fact, suppose that $x_{*} \in P_{h}$ is another fixed point of operator $A$. Let

$$
c_{1}=\sup \left\{0<c \leq 1 \mid c x_{*} \leq x^{*} \leq \frac{1}{c} x_{*}\right\} .
$$

Clearly, $0<c_{1} \leq 1$ and $c_{1} x_{*} \leq x^{*} \leq\left(1 / c_{1}\right) x_{*}$. If $0<c_{1}<1$, according to $\left(C_{1}\right)$, there exists $t_{3} \in(a, b)$ such that $\tau\left(t_{3}\right)=c_{1}$. Then

$$
\begin{aligned}
x^{*} & =A\left(x^{*}, x^{*}\right) \geq A\left(c_{1} x_{*}, \frac{1}{c_{1}} x_{*}\right)=A\left(\tau\left(t_{3}\right) x_{*}, \frac{1}{\tau\left(t_{3}\right)} x_{*}\right) \\
& \geq \varphi\left(t_{3}\right) A\left(x_{*}, x_{*}\right)=\varphi\left(t_{3}\right) x_{* \prime} \\
x^{*} & =A\left(x^{*}, x^{*}\right) \leq A\left(\frac{1}{c_{1}} x_{*}, c_{1} x_{*}\right)=A\left(\frac{1}{\tau\left(t_{3}\right)} x_{*}, \tau\left(t_{3}\right) x_{*}\right) \\
& \leq \frac{1}{\varphi\left(t_{3}\right)} A\left(x_{*}, x_{*}\right)=\frac{1}{\varphi\left(t_{3}\right)} x_{*} .
\end{aligned}
$$

It follows that

$$
\varphi\left(t_{3}\right) x_{*} \leq x^{*} \leq \frac{1}{\varphi\left(t_{3}\right)} x_{*} .
$$


Hence, $c_{1} \geq \varphi\left(t_{3}\right)>\tau\left(t_{3}\right)=c_{1}$, which is a contradiction. Thus we have $c_{1}=1$, that is, $x_{*}=x^{*}$. Therefore, $A$ has a unique fixed point $x^{*}$ in $P_{h}$. Note that $\left[u_{0}, v_{0}\right] \subset P_{h}$, so we know that $x^{*}$ is the unique fixed point of $A$ in $\left[u_{0}, v_{0}\right]$. For any initial $x_{0}, y_{0} \in P_{h}$, we can choose a small number $\bar{e} \in(0,1)$ such that

$$
\bar{e} h \leq x_{0} \leq \frac{1}{\bar{e}} h, \quad \bar{e} h \leq y_{0} \leq \frac{1}{\bar{e}} h .
$$

From $\left(C_{1}\right)$, there is $t_{4} \in(a, b)$ such that $\tau\left(t_{4}\right)=\bar{e}$, thus

$$
\tau\left(t_{4}\right) h \leq x_{0} \leq \frac{1}{\tau\left(t_{4}\right)} h, \quad \tau\left(t_{4}\right) h \leq y_{0} \leq \frac{1}{\tau\left(t_{4}\right)} h .
$$

We can choose a sufficiently large positive integer $q$ such that

$$
\left(\frac{\varphi\left(t_{4}\right)}{\tau\left(t_{4}\right)}\right)^{q} \geq \frac{1}{\tau\left(t_{4}\right)}
$$

Take $\widehat{u}_{0}=\left[\tau\left(t_{4}\right)\right]^{q} h, \widehat{v}_{0}=\left(1 /\left[\tau\left(t_{4}\right)\right]^{q}\right) h$. We can find that

$$
\widehat{u}_{0} \leq x_{0} \leq \widehat{v}_{0}, \quad \widehat{u}_{0} \leq y_{0} \leq \widehat{v}_{0},
$$

constructing successively the sequences

$$
\begin{array}{lll}
x_{n}=A\left(x_{n-1}, y_{n-1}\right), & y_{n}=A\left(y_{n-1}, x_{n-1}\right), & n=1,2, \ldots, \\
\widehat{u}_{n}=A\left(\widehat{u}_{n-1}, \widehat{v}_{n-1}\right), & \widehat{v}_{n}=A\left(\widehat{v}_{n-1}, \widehat{u}_{n-1}\right), & n=1,2, \ldots
\end{array}
$$

By using the mixed monotone properties of operator $A$, we have

$$
\widehat{u}_{n} \leq x_{n} \leq \widehat{v}_{n}, \quad \widehat{u}_{n} \leq y_{n} \leq \widehat{v}_{n}, \quad n=1,2, \ldots
$$

Similarly to the above proof, we can know that there exists $y^{*} \in P_{h}$ such that

$$
A\left(y^{*}, y^{*}\right)=y^{*}, \quad \lim _{n \rightarrow \infty} \widehat{u}_{n}=\lim _{n \rightarrow \infty} \widehat{v}_{n}=y^{*}
$$

By the uniqueness of fixed points of operator $A$ in $P_{h}$, we have $y^{*}=x^{*}$. Taking into account that $P$ is normal, we deduce that $\lim _{n \rightarrow \infty} x_{n}=\lim _{n \rightarrow \infty} y_{n}=x^{*}$. This completes the proof.

\section{Applications to Singular BVP (1.1) on Time Scales}

A Banach space $E=C\left(\left[a, \sigma^{2}(b)\right]_{\mathbb{T}}\right)$ is the set of real-valued continuous (in the topology of $\mathbb{T}$ ) function $u(t)$ defined on $\left[a, \sigma^{2}(b)\right]_{\mathbb{T}}$ with the norm $\|u\|=\max _{t \in\left[a, \sigma^{2}(b)\right]_{\mathbb{T}}}|u(t)|$. 
Define a cone by

$$
P=\left\{u \in E \mid \min _{t \in\left[a, \sigma^{2}(b)\right]_{\mathbb{T}}} u(t) \geq 0\right\}
$$

It is clear that $P$ is a normal cone of which the normality constant is 1 .

In order to obtain our main result, we need the following lemmas.

Lemma 3.1 (see [18]). The Green function corresponding to the following problem

$$
\begin{gathered}
-x^{\Delta \Delta}(t)=0, \quad t \in[a, b]_{\mathbb{T}}, \\
x(a)=0, \quad x\left(\sigma^{2}(b)\right)=\delta x(\eta)
\end{gathered}
$$

is given by

$$
K(t, s)=G(t, s)+\frac{\delta G(\eta, s)}{\sigma^{2}(b)-a-\delta(\eta-a)}(t-a)
$$

where

$$
G(t, s)=\frac{1}{\sigma^{2}(b)-a} \begin{cases}(t-a)\left(\sigma^{2}(b)-\sigma(s)\right), & t \leq s, \\ (\sigma(s)-a)\left(\sigma^{2}(b)-t\right), & t \geq \sigma(s),\end{cases}
$$

is Green's function for the BVP:

$$
\begin{gathered}
-x^{\Delta \Delta}(t)=0, \quad t \in[a, b]_{\mathbb{T}}, \\
x(a)=x\left(\sigma^{2}(b)\right)=0 .
\end{gathered}
$$

Lemma 3.2 (see $[18])$. For any $(t, s) \in\left[a, \sigma^{2}(b)\right]_{\mathbb{T}} \times[a, \sigma(b)]_{\mathbb{T}}$, we have

$$
\frac{\delta G(\eta, s)}{\sigma^{2}(b)-a-\delta(\eta-a)}(t-a) \leq K(t, s) \leq\left[1+\frac{\delta G(\eta, s)}{\sigma^{2}(b)-a-\delta(\eta-a)}\right](t-a) .
$$

Our main result is the following theorem.

Theorem 3.3. Assume that

$\left(E_{1}\right) f_{1}$ is nondecreasing, $f_{2}$ is nonincreasing and there exist $\tau(t), \varphi(t)$ on interval $(a, b)_{\mathbb{T}}$ such that $\tau:(a, b)_{\mathbb{T}} \rightarrow(0,1)$ is a surjection and $\varphi(t)>\tau(t)$, for all $t \in(a, b)_{\mathbb{T}}$ which satisfy

$$
f_{1}\left(\tau(\mu) x_{1}\right)+f_{2}\left(\frac{1}{\tau(\mu)} x_{2}\right) \geq \varphi(\mu)\left[f_{1}\left(x_{1}\right)+f_{2}\left(x_{2}\right)\right], \quad \forall \mu \in(a, b)_{\mathbb{T}}, x_{1}, x_{2} \in P
$$


$\left(E_{2}\right)$ there exist two constants $N_{1}, N_{2}>0$ and $h \in P \backslash\{0\}$ such that

$$
N_{1} h(t) \leq \int_{a}^{\sigma(b)} K(t, s) w(s)\left[f_{1}(h(s))+f_{2}(h(s))\right] \Delta s \leq N_{2} h(t), \quad \forall t \in\left[a, \sigma^{2}(b)\right]_{\mathbb{T}} .
$$

Then problem (1.1) has a unique positive solution $x^{*}$ in $P_{h}$. Moreover, for any initial $x_{0}, y_{0} \in P_{h}$, constructing successively the sequences

$$
\begin{aligned}
x_{n}(t)= & \int_{a}^{\sigma(b)} K(t, s) w(s)\left[f_{1}\left(x_{n-1}(s)\right)+f_{2}\left(y_{n-1}(s)\right)\right] \Delta s, \quad t \in\left[a, \sigma^{2}(b)\right]_{\mathbb{T}^{\prime}} n=1,2, \ldots, \\
y_{n}(t)= & \int_{a}^{\sigma(b)} K(t, s) w(s)\left[f_{1}\left(y_{n-1}(s)\right)+f_{2}\left(x_{n-1}(s)\right)\right] \Delta s, \quad t \in\left[a, \sigma^{2}(b)\right]_{\mathbb{T}^{\prime}} n=1,2, \ldots, \\
& \text { we have }\left\|x_{n}-x^{*}\right\| \rightarrow 0 \text { and }\left\|y_{n}-x^{*}\right\| \rightarrow 0 \text { as } n \rightarrow \infty .
\end{aligned}
$$

Proof of Theorem 3.3. Define an operator $A: P \times P \rightarrow E$

$$
A\left(x_{1}, x_{2}\right)(t)=\int_{a}^{\sigma(b)} K(t, s) w(s)\left[f_{1}\left(x_{1}(s)\right)+f_{2}\left(x_{2}(s)\right)\right] \Delta s, \quad t \in\left[a, \sigma^{2}(b)\right]_{\mathbb{T}} .
$$

It is easy to check that $x$ is a solution of problem (1.1) if and only if $x$ is a fixed point of operator $A$. Clearly, we can know that $A: P \times P \rightarrow P$ is a mixed monotone operator. For any $\mu \in(a, b)_{\mathbb{T}}$ and $x_{1}, x_{2} \in P$, according to $\left(E_{1}\right)$, we obtain

$$
\begin{aligned}
A\left(\tau(\mu) x_{1}, \frac{1}{\tau(\mu)} x_{2}\right) & =\int_{a}^{\sigma(b)} K(t, s) w(s)\left[f_{1}\left(\tau(\mu) x_{1}(s)\right)+f_{2}\left(\frac{1}{\tau(\mu)} x_{2}(s)\right)\right] \Delta s \\
& \geq \varphi(\mu) \int_{a}^{\sigma(b)} K(t, s) w(s)\left[f_{1}\left(x_{1}(s)\right)+f_{2}\left(x_{2}(s)\right)\right] \Delta s \\
& =\varphi(\mu) A\left(x_{1}, x_{2}\right) .
\end{aligned}
$$

Hence,

$$
A\left(\tau(\mu) x_{1}, \frac{1}{\tau(\mu)} x_{2}\right) \geq \varphi(\mu) A\left(x_{1}, x_{2}\right), \quad \text { for } \mu \in(a, b)_{\mathbb{T}}, x_{1}, x_{2} \in P
$$

In addition, from $\left(E_{2}\right)$, we know that

$$
N_{1} h(t) \leq A(h, h)=\int_{a}^{\sigma(b)} K(t, s) w(s)\left[f_{1}(h(s))+f_{2}(h(s))\right] \Delta s \leq N_{2} h(t), \quad \forall t \in\left[a, \sigma^{2}(b)\right]_{\mathbb{T}} .
$$

Thus $A(h, h) \in P_{h}$. Therefore, all the conditions of Theorem 2.3 are satisfied. By Theorem 2.3, we can obtain the conclusions of Theorem 3.3. 
Now, let us end this paper by the following example.

Example 3.4. Let $\mathbb{T}=[0,1 / 2] \cup[2 / 3,1]$, consider the following BVP on time scales

$$
\begin{gathered}
-x^{\Delta \Delta}(t)=\frac{1}{t}\left[3+x^{1 / 2}(t)+5+\frac{1}{x^{1 / 2}(t)}\right], \quad t \in[0,1]_{\mathbb{T}}, \\
x(0)=0, \quad x(1)=2 x\left(\frac{1}{3}\right) .
\end{gathered}
$$

Set $w(t)=1 / t, f_{1}(x)=3+x^{1 / 2}, f_{2}(x)=5+1 / x^{1 / 2}, \tau(t)=t, \varphi(t)=t^{2 / 3}$. Then $\tau:(0,1)_{\mathbb{T}} \rightarrow(0,1)$ is a surjection and $\varphi(t)>\tau(t)$ for $t \in(0,1)_{\mathbb{T}}$.

For any $t \in(0,1), x_{1}, x_{2} \in P$, it is easy to check that

$$
\begin{aligned}
f_{1}\left(t x_{1}\right)+f_{2}\left(\frac{1}{t} x_{2}\right) & =3+\left(t x_{1}\right)^{1 / 2}+5+\frac{1}{\left(x_{2} / t\right)^{1 / 2}} \\
& \geq t^{2 / 3}\left(3+x_{1}^{1 / 2}+5+\frac{1}{x_{2}^{1 / 2}}\right) \\
& =t^{2 / 3}\left[f_{1}\left(x_{1}\right)+f_{2}\left(x_{2}\right)\right] .
\end{aligned}
$$

It follows from Lemma 3.1 that

$$
G\left(\frac{1}{3}, s\right)= \begin{cases}\frac{1}{3}(1-\sigma(s)), & \frac{1}{3} \leq s \\ \frac{2}{3} \sigma(s), & \frac{1}{3} \geq \sigma(s) .\end{cases}
$$

Let $\int_{0}^{1} 6 G(1 / 3, s)(1 / s)\left(3+s^{1 / 2}+5+1 / s^{1 / 2}\right) \Delta s=d$, since

$$
\begin{aligned}
d= & 6 \int_{0}^{1 / 3} \frac{2}{3} s \frac{1}{s}\left(8+s^{1 / 2}+\frac{1}{s^{1 / 2}}\right) d s+6 \int_{1 / 3}^{1 / 2} \frac{1}{3}(1-s) \frac{1}{s}\left(8+s^{1 / 2}+\frac{1}{s^{1 / 2}}\right) d s \\
& +6 \int_{1 / 2}^{2 / 3} \frac{1}{3}(1-\sigma(s)) \frac{1}{s}\left(8+s^{1 / 2}+\frac{1}{s^{1 / 2}}\right) \Delta s+6 \int_{2 / 3}^{1} \frac{1}{3}(1-\sigma(s)) \frac{1}{s}\left(8+s^{1 / 2}+\frac{1}{s^{1 / 2}}\right) d s \\
= & 32(\ln 3-\ln 2)-\frac{34}{9}\left(\frac{1}{2}\right)^{-1 / 2}-\frac{4}{3}\left(\frac{1}{2}\right)^{3 / 2}+4\left(\frac{1}{3}\right)^{-1 / 2} \\
& +16\left(\frac{1}{3}\right)^{5 / 2}+\frac{2}{9}\left(\frac{1}{2}\right)^{1 / 2}+4\left(\frac{2}{3}\right)^{-1 / 2}+8\left(\frac{1}{3}\right)^{1 / 2}-\frac{8}{9}
\end{aligned}
$$

$>0$. 
We choose $h(t)=t-a=t$, according to Lemma 3.2, we have

$$
\begin{gathered}
\int_{0}^{1} K(t, s) \frac{1}{s}\left[f_{1}(s)+f_{2}(s)\right] \Delta s \geq t d \\
\int_{0}^{1} K(t, s) \frac{1}{s}\left[f_{1}(s)+f_{2}(s)\right] \Delta s \leq t\left[d+\int_{0}^{1} \frac{1}{s}\left(8+s^{1 / 2}+\frac{1}{s^{1 / 2}}\right) \Delta s\right] .
\end{gathered}
$$

By Theorem 3.3, problem (3.14) has a unique positive solution $x^{*}$ in $P_{h}=P_{t}$. For any initial $x_{0}, y_{0} \in P_{t}$, constructing successively the sequences

$$
\begin{aligned}
& x_{n}(t)=\int_{0}^{1} K(t, s) \frac{1}{s}\left[8+x_{n-1}^{1 / 2}(s)+\frac{1}{y_{n-1}^{1 / 2}(s)}\right] \Delta s, \quad t \in[0,1]_{\mathbb{T}}, n=1,2, \ldots, \\
& y_{n}(t)=\int_{0}^{1} K(t, s) \frac{1}{s}\left[8+y_{n-1}^{1 / 2}(s)+\frac{1}{x_{n-1}^{1 / 2}(s)}\right] \Delta s, \quad t \in[0,1]_{\mathbb{T}}, n=1,2, \ldots,
\end{aligned}
$$

we have $\left\|x_{n}-x^{*}\right\| \rightarrow 0,\left\|y_{n}-x^{*}\right\| \rightarrow 0$ as $n \rightarrow \infty$.

Remark 3.5. Example 3.4 indicates that Theorem 3.3 generalizes and complements Theorem 2.3 in [18] at the following aspects. Firstly, in our proof, we only need to check the conditions "there exists $h \in P \backslash\{\theta\}$ such that $A(h, h) \in P_{h}$ ", in fact, the author has shown that "A: $P_{h} \rightarrow P_{h}$ " in the proof of Theorem 2.3 in [18]. It is clear that our hypotheses are weaker than those imposed in Theorem 2.3 in [18]. According to Lemma 3.2, we can know that the condition $\left(E_{2}\right)$ is automatically satisfied. Secondly, we have considered the case that the condition " $\tau(t)=t$ and $\varphi(t)=t^{q}(q \in(0,1))$ " is not satisfied, therefore, the condition $\left(E_{1}\right)$ incorporates the more comprehensive functions than the condition $\left(H_{3}\right)$ in Theorem 2.3 in [18]. Thirdly, the more general conditions are imposed on our nonlinear term, they can be the sum of nondecreasing functions and nonincreasing functions.

\section{Acknowledgment}

H. Xu was supported financially by the Science Foundation of North University of China.

\section{References}

[1] D. Guo and V. Lakshmikantham, "Coupled fixed points of nonlinear operators with applications," Nonlinear Analysis: Theory, Methods E Applications, vol. 11, no. 5, pp. 623-632, 1987.

[2] D. J. Guo and V. Lakshmikantham, Nonlinear Problems in Abstract Cones, vol. 5 of Notes and Reports in Mathematics in Science and Engineering, Academic Press, Boston, Mass, USA, 1988.

[3] Z. Q. Zhao, "Existence and uniqueness of fixed points of some mixed monotone mappings in partially ordered linear spaces," Journal of Systems Science and Mathematical Sciences, vol. 19, no. 2, pp. 217-224, 1999 (Chinese).

[4] D. J. Guo, Partial Order Methods in Nonlinear Analysis, Shandong Science and Technology Press, Jinan, China, 2000.

[5] Y. X. Wu and Z. D. Liang, "Existence and uniqueness of fixed points for mixed monotone operators with applications," Nonlinear Analysis: Theory, Methods \& Applications, vol. 65, no. 10, pp. 1913-1924, 2006. 
[6] Z. Drici, F. A. McRae, and J. V. Devi, "Fixed point theorems for mixed monotone operators with PPF dependence," Nonlinear Analysis: Theory, Methods E Applications, vol. 69, no. 2, pp. 632-636, 2008.

[7] Z. Zhang and K. Wang, "On fixed point theorems of mixed monotone operators and applications," Nonlinear Analysis: Theory, Methods E Applications, vol. 70, no. 9, pp. 3279-3284, 2009.

[8] Z. Q. Zhao, "Existence and uniqueness of fixed points for some mixed monotone operators," Nonlinear Analysis: Theory, Methods \& Applications, vol. 73, no. 6, pp. 1481-1490, 2010.

[9] C.-B. Zhai and X.-M. Cao, "Fixed point theorems for $\tau-\varphi$-concave operators and applications," Computers $\mathcal{E}$ Mathematics with Applications, vol. 59, no. 1, pp. 532-538, 2010.

[10] F. M. Atici and G. S. Guseinov, "On Green's functions and positive solutions for boundary value problems on time scales," Journal of Computational and Applied Mathematics, vol. 141, no. 1-2, pp. 75-99, 2002.

[11] M. Bohner and A. Peterson, Dynamic Equations on Time Scales: An Introduction with Applications, Birkhäuser, Boston, Mass, USA, 2001.

[12] M. Bohner and A. Peterson, Eds., Advances in Dynamic Equations on Time Scales, Birkhäuser, Boston, Mass, USA, 2003.

[13] D. R. Anderson, "Solutions to second-order three-point problems on time scales," Journal of Difference Equations and Applications, vol. 8, no. 8, pp. 673-688, 2002.

[14] D. R. Anderson and P. J. Y. Wong, "Positive solutions for second-order semipositone problems on time scales," Computers \& Mathematics with Applications, vol. 58, no. 2, pp. 281-291, 2009.

[15] D. R. Anderson and C. Zhai, "Positive solutions to semi-positone second-order three-point problems on time scales," Applied Mathematics and Computation, vol. 215, no. 10, pp. 3713-3720, 2010.

[16] E. R. Kaufmann, "Positive solutions of a three-point boundary-value problem on a time scale," Electronic Journal of Differential Equations, vol. 82, pp. 1-11, 2003.

[17] N. A. Hamal and F. Yoruk, "Positive solutions of nonlinear $m$-point boundary value problems on time scales," Journal of Computational and Applied Mathematics, vol. 231, no. 1, pp. 92-105, 2009.

[18] J.-P. Sun, "Existence of positive solution to second-order three-point BVPs on time scales," Boundary Value Problems, vol. 2009, Article ID 685040, 6 pages, 2009.

[19] S. Hilger, "Analysis on measure chains-a unified approach to continuous and discrete calculus," Results in Mathematics. Resultate der Mathematik, vol. 18, no. 1-2, pp. 18-56, 1990.

[20] V. Jamieson and V. Spedding, "Taming nature's numbers," New Scientist: The Global Science and Technology Weekly, vol. 2404, pp. 28-31, 2003.

[21] F. M. Atici, D. C. Biles, and A. Lebedinsky, "An application of time scales to economics," Mathematical and Computer Modelling, vol. 43, no. 7-8, pp. 718-726, 2006.

[22] C. C. Tisdell and A. Zaidi, "Basic qualitative and quantitative results for solutions to nonlinear, dynamic equations on time scales with an application to economic modelling," Nonlinear Analysis: Theory, Methods \& Applications, vol. 68, no. 11, pp. 3504-3524, 2008.

[23] J. J. DaCunha, J. M. Davis, and P. K. Singh, "Existence results for singular three point boundary value problems on time scales," Journal of Mathematical Analysis and Applications, vol. 295, no. 2, pp.378-391, 2004.

[24] M. Bohner and H. Luo, "Singular second-order multipoint dynamic boundary value problems with mixed derivatives," Advances in Difference Equations, vol. 2006, Article ID 54989, 15 pages, 2006.

[25] Z.-C. Hao, T.-J. Xiao, and J. Liang, "Existence of positive solutions for singular boundary value problem on time scales," Journal of Mathematical Analysis and Applications, vol. 325, no. 1, pp. 517-528, 2007.

[26] H. Luo, "Positive solutions to singular multi-point dynamic eigenvalue problems with mixed derivatives," Nonlinear Analysis: Theory, Methods \& Applications, vol. 70, no. 4, pp. 1679-1691, 2009.

[27] L. G. Hu, "Positive solutions to singular third-order three-point boundary value problems on time scales," Mathematical and Computer Modelling, vol. 51, no. 5-6, pp. 606-615, 2010. 